
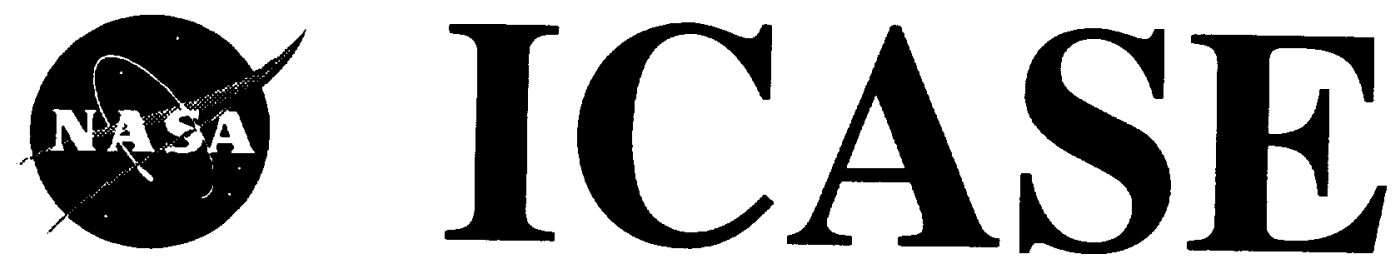

\title{
A PHENOMENOLOGICAL TREATMENT OF ROTATING TURBULENCE
}

\section{Ye Zhou}

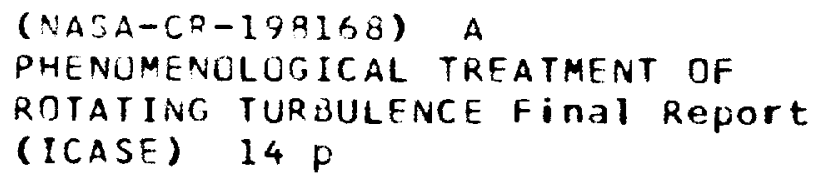

N95-28228

Unclas

$63 / 34 \quad 0051396$

Contract No. NAS1-19480

May 1995

Institute for Computer Applications in Science and Engineering NASA Langley Research Center

Hampton, VA 23681-0001

SRA Operated by Universities Space Research Association 
$\sim r$ 


\title{
A PHENOMENOLOGICAL TREATMENT OF ROTATING TURBULENCE
}

\author{
Ye Zhou* \\ Institute for Computer Applications in Science and Engineering \\ NASA Langley Research Center, Hampton, VA 23681-0001
}

\begin{abstract}
The strong similarity between the magnetohydrodynamic (MHD) turbulence and initially isotropic turbulence subject to rotation is noted. We then apply the MHD phenomenologies of Kraichnan and Matthaeus \& Zhou to rotating turbulence. When the turbulence is subject to a strong rotation, the energy spectrum is found to scale as $E(k)=C_{\Omega}(\Omega \epsilon)^{1 / 2} k^{-2}$, where $\Omega$ is the rotation rate, $k$ is the wavenumber, and $\epsilon$ is the dissipation rate. This spectral form is consistent with a recent letter by Zeman. However, here the constant $C_{\Omega}$ is found to be related to the Kolmogorov constant and is estimated in the range $1.22-1.87$ for the typical values of the latter constant. A 'rule' that relates spectral transfer times to the eddy turnover time and the time scale for decay of the triple correlations is deduced. A hypothesis for the triple correlation decay rate leads to the spectral law which varies between the ' $-5 / 3$ ' (without rotation) and ' -2 ' laws (with strong rotation). For intermediate rotation rates, the spectrum varies according to the value of a dimensionless parameter that measures the strength of the rotation wavenumber $k_{\Omega}=\left(\Omega^{3} / \epsilon\right)^{1 / 2}$ relative to the wavenumber $k$. An eddy viscosity is derived with an explicit dependence on the rotation rate.
\end{abstract}

*This research was supported by the National Aeronautics and Space Administration under NASA Contract No. NAS1-19480 while the author was in residence at the Institute for Computer Applications in Science and Engineering (ICASE), NASA Langley Research Center, Hampton, VA 23681-0001. 



\section{Introduction}

For homogeneous, isotropic, statistically steady turbulence, the classical Kolmogorov phenomenology leads to the well known energy spectrum

$$
E(k)=C_{K} \epsilon^{2 / 3} k^{-5 / 3}
$$

Here $\epsilon$ is the energy dissipation rate, $k$ is the wavenumber, and $C_{K}$ is the Kolmogorov constant. The Kolmogorov hypotheses implies that the energy-containing range excitation does not affect energy transfer within the inertial range. Therefore, the average rate of energy dissipation is identified with the rate of spectral energy transfer and the rate of energy input. The nonlinear (or eddy turnover) time-scale, $\tau_{n l}(k) \equiv\left[k^{3} E(k)\right]^{-1}$, is then equivalent to the spectral transfer time, $\tau_{s}\left(\right.$ Kolmogorov $^{1} ;$ Batchelor $^{2}$; Monin and Yaglom $^{3}$ ).

In this report, attention is called to the strong similarity between the magnetohydrodynamic (MHD) turbulence and isotropic turbulence subject to solid body rotation. For MHD turbulence, Kraichnan ${ }^{4}$ argued that the propagation of the Alfvénic fluctuations disrupts phase relation and thereby may be expected, on the average, to decrease energy transfer. Experimental studies ${ }^{5-8}$, direct numerical simulations (DNS) ${ }^{9-12}$, large-eddy simulations $(\mathrm{LES})^{9,13}$, and closure approximations ${ }^{14}$ have established that solid body rotation suppresses the nonlinear energy cascade from large scale to small scale. Specifically, uniform rotation causes plane waves to propagate with phase speed $2 \Omega k_{z} / k$. Here the rotation vector is considered to act along the vertical $\left(\frac{z}{|z|}\right)$. The effect of rotation on the spectral transfer is through phase scrambling. Based on this evidence, the phenomenology $y^{4,15}$ originally developed for MHD turbulence is applied to rotating turbulence.

\section{Energy Spectra and Transfer}

The Kolmogorov's phenomenology cannot be simply extended to more complex situations which may be governed by other time-scales. Special generalizations of the theory are nec- 
essary. Kraichnan ${ }^{4}$ generalized the Kolmogorov theory for MHD turbulence so that the influence of the Alfvén wave on the MHD spectrum can be included. In addition to the nonlinear time scale, there is a much shorter Alfvén time scale $\tau_{A}=\left[k B_{0}\right]^{-1}$, where $B_{0}$ is the characteristic magnetic field strength in Alfvén speed unit. In order to infer the form of the inertial-range spectrum, it is necessary to estimate the magnitude for the triple correlations. If the energy-containing range excitations were absent, it would be expected that the nonlinear interaction would build up substantial triple correlations in the local dynamical time $\tau_{n l}(k)$. With the energy-containing excitation present, however, the Alfvén time scale $\tau_{A}$ is the effective time for relaxation of the locally built-up phase correlations through propagation. In general, $\tau_{3}$, the time scale for decay of triple correlations which is responsible for inducing turbulent spectral transfer, may depend on any relevant turbulence parameters. Because energy is conserved by the nonlinear interaction and a local cascade has been assumed, $\epsilon$ is independent of $k$. Local cascade also implies that $\epsilon$ is explicitly proportional to $\tau_{3}$ and depends on the wavenumber and on power of the omni-directional energy spectrum ${ }^{4}$. A simple dimensional analysis leads to

$$
\epsilon=A^{2} \tau_{3}(k) k^{4} E^{2}(k)
$$

where $A$ is a constant. In steady state isotropic turbulence, the only time scale available for $\tau_{3}(k)$ is the nonlinear time scale, $\tau_{n l}(k)$; therefore the Kolmogorov ${ }^{1}$ spectrum is recovered. Using $\tau_{3}(k)=\tau_{A}(k)$, Kraichnan ${ }^{4}$ obtained the MHD energy spectrum

$$
E(k) \propto\left(B_{0} \epsilon\right)^{1 / 2} k^{-3 / 2}
$$

Recently, Squires et al. ${ }^{13}$ extended one point analysis of the long-time evolution of kinetic energy and length scales to rotating homogeneous turbulence. In regime of high Reynolds numbers and low Rossby numbers, rotating turbulence is characterized by two disparate timescales: a short timescales associated the rotating frequency $\tau_{\Omega}=1 / \Omega$ and a nonlinear time scale. Squires et al. ${ }^{13}$ assumed that the correlation time of the nonlinear triadic interactions 
is directly proportional to the short time scale $\tau_{\Omega}$. The predictions of the asymptotic decay for the kinetic energy in rotating turbulence, based on this crucial assumption on the time scale, have been confirmed within a few percent by their LES runs for times of order $O\left(10^{3}\right)$ of initial turbulence. A direct application of $\tau_{3}=\tau_{\Omega}$ produces the following energy spectrum for turbulence subject to strong rotation:

$$
E(k)=C_{\Omega}(\Omega \epsilon)^{1 / 2} k^{-2}
$$

where $C_{\Omega}$ is a constant. The spectral form in Eq. (4) is consistent with a recent letter of Zeman $^{16}$ which motived present study. However, here we will determine the constant $C_{\Omega}$.

For steady isotropic turbulence, the wavenumber dependent spectral transfer time $\tau_{s}(k)$ can be defined through $\epsilon=U_{k}^{2} / \tau_{s}(k)$, where $U_{k}=[k E(k)]^{1 / 2}$ is the characteristic velocity of eddies at wavenumber $k$ and $\epsilon$ is the steady, wavenumber-independent energy transfer rate. Using (2) and the definition of $\tau_{n l}$ and $U_{k}$, it was found that

$$
\tau_{s}(k)=\frac{1}{A^{2}} \frac{\left[\tau_{n l}(k)\right]^{2}}{\tau_{\Omega}(k)} .
$$

A corollary to this development is the time for the spectral energy transfer time is increased to a value greater than $\tau_{n l}$; thus the nonlinear energy transfer is suppressed by the rotation. Similar suggestion has been made for MHD turbulence ${ }^{17-18}$.

For sufficiently large Reynolds numbers for which a universal equilibrium exists, the expression for the skewness

$$
S_{3}=-\frac{3(30)^{1 / 2}}{7} \nu \frac{\int_{0}^{\infty} k^{4} E(k) d k}{\left[\int_{0}^{\infty} k^{2} E(k) d k\right]^{3 / 2}}
$$

is exact (Batchelor ${ }^{2}$, Monin and Yaglom ${ }^{3}$ ). Eqs. (4) and (6) implies that the skewness factors of the spatial derivatives of the fields approach to zero as the rotation rate go to infinity $\left(S_{3} \propto 1 / \Omega^{1 / 4}\right)$. From the single point modeling perspective ${ }^{19}$, the reduction of the skewness leads to the decreasing of the production term in the dissipation equation.

Following the MHD development, we make use of the viewpoint that the lifetime of triple correlations in rotating turbulence might be more accurately treated by taking into 
account the possibility that these correlations decay because of the influence of both wave propagation and nonlinear triadic interactions (Matthaeus and Zhou ${ }^{15}$ ). The simple choice

$$
\frac{1}{\tau_{3}(k)}=\frac{1}{\tau_{n l}(k)}+\frac{1}{\tau_{\Omega}(k)}
$$

satisfies the appropriate limiting cases: $\tau_{3}(k) \rightarrow \tau_{n l}$ without rotation and $\tau_{3}(k) \rightarrow \tau_{\Omega}$ with strong rotation. The most general 'rule' is

$$
\tau_{s}(k)=\frac{1}{A^{2}} \frac{\tau_{n l}^{2}(k)}{\tau_{3}(k)} .
$$

Substituting (7) into (2), the generalized inertial range energy spectrum is

$$
E(k)=Z^{2} A^{-4 / 3} \epsilon^{2 / 3} k^{-5 / 3}
$$

where $Z$ is the solution of the dimensionless equation

$$
Z^{4}=Z_{0}+Z
$$

where

$$
Z_{0}=A^{2 / 3} \frac{\Omega}{\left(\epsilon k^{2}\right)^{1 / 3}}=\left[\frac{A k_{\Omega}}{k}\right]^{2 / 3} .
$$

The parameter $k_{\Omega}=\left(\Omega^{3} / \epsilon\right)^{1 / 2}$ has appeared in Zeman ${ }^{16}$. Eqs. (9)-(11) reduce to the nonrotating Kolmogorov "-5/3" spectrum when $Z_{0} \rightarrow 0$ (so that $Z \rightarrow 1$ ), and to our rotating modified "-2" spectrum when $Z_{0} \rightarrow \infty$ (so that $Z \rightarrow Z_{0}^{1 / 4}$ ). As shown in Matthaeus and Zhou ${ }^{15}$, Eqs. (10)-(11) have only one physically acceptable solution to this quartic equation:

$$
Z=\frac{1}{2}\left(\sqrt{Y}+\sqrt{-Y+2 \sqrt{Y^{2}+4 Z_{0}}}\right)
$$

where

$$
Y=\sqrt[3]{\frac{1}{2}+\sqrt{\frac{1}{4}+\left(\frac{4 Z_{0}}{3}\right)^{3}}}+\sqrt[3]{\frac{1}{2}-\sqrt{\frac{1}{4}+\left(\frac{4 Z_{0}}{3}\right)^{3}}} .
$$

From Eqs. (9)-(11), the constant of the rotating spectrum can be estimated. Taking the Kolmogorov limit, we find that $A=C_{K}^{-3 / 4}$ (Matthaeus and Zhou ${ }^{15}$ ). The strong rotation limit then leads to $C_{\Omega}=1 / A=1.22-1.87$ for the typical range of Kolmogorov constant. Fig. 
1 illustrates spectra obtained this way with $\epsilon=1$ and $A=0.7$ (we have chosen $C_{K}=1.5$ ), varying the reference rotation rate over values $\Omega=0,10$, and 100 . The zero rotation case is a pure Kolmogorov spectrum while the $\Omega=100$ case is very nearly $k^{-2}$ spectrum (Eq.4). For intermediate rotation rates the spectrum varies smoothly between these two limiting forms, according to the increase of the controlling parameter $Z_{0}$ with increasing ratio $k_{\Omega} / k$.

\section{Eddy Viscosity}

The rotation dependence eddy viscosity can be estimated using Zeman's procedure ${ }^{16}$. Following Tennekes and Lumley ${ }^{20}$, it is reasonable to assume that in the inertial range

$$
T(k)=\epsilon \propto \nu_{T}\left(k^{\prime \prime}\right) S^{2}\left(k^{\prime}\right)
$$

where, approximately, $k / 3<k^{\prime} \leq k$ and $k^{\prime \prime}=3 k^{\prime}$, and $S(k)=1 / \tau_{n l}=\left[k^{3} E(k)\right]^{1 / 2}$. (Ref. 21 verified the predictions of Tennekes and Lumley ${ }^{20}$ on the scaling laws of the energy transfer) Using (2), the spectral eddy viscosity is

$$
\nu_{T}(k)=\nu_{0}\left[\frac{E(k)}{k}\right]^{1 / 2} \frac{1}{1+y^{-1 / 2}}
$$

where $\nu_{0}$ is a constant and $y=k^{3} E(k) / \Omega^{2}$, following Zeman ${ }^{16}$. Equation (15a) is expected to find use in the spectral LES of rotating turbulence.

The inertial range wavenumber $k$ can be related ${ }^{22-24}$ to the turbulent kinetic energy $K$ and dissipation rate $\epsilon$

$$
k=\frac{E(k)}{K^{3 / 2}}\left(3 C_{k} / 2\right)^{3 / 2}
$$

For the inertial range spectrum, Eq. (15a) can be rewritten in physical space:

$$
\nu_{T}(x)=\nu_{0}^{\prime} \frac{K^{2}}{\epsilon} \frac{1}{1+0.36 K \Omega / \epsilon},
$$

The eddy viscosity form (15b) is only appropriate for homogeneous turbulence without mean velocity gradients. For turbulent flows with mean velocity gradients in a rotating frame, the effect of rotations appears in conjunction with the mean vorticity (Speziale ${ }^{25}$ ) and Eq. 
(15b) can be extended in a straightforward way by replacing $\Omega$ by $\left(\bar{W}_{i j} \bar{W}_{i j}\right)^{1 / 2}$ (Gatski and Speziale $\left.{ }^{26}\right)$. Here $\bar{W}_{i j}=\bar{\omega}_{i j}+\alpha \epsilon_{m j i} \Omega_{m}$ is the extended absolute vorticity tensor defined in

Gatski and Speziale ${ }^{26}$ ( $\alpha$ is a constant). The constant $\nu_{0}^{\prime}$ can then be evaluated in homogeneous shear flow where $W_{i j}$ reduces to the uniform shear rate. Such flows are deferred to a future study.

\section{Discussion}

These results are now compared to Zeman ${ }^{16}$. He started with a simplified Lagrangian description of the relation between stress and mean strain in rotating turbulence and proposed the modified the inertial range spectrum when $\tau_{\Omega} \gg \tau_{n l}$

$$
E(k) \propto \epsilon^{2 / 5} \Omega^{4 / 5} k^{-11 / 5}
$$

Zeman's generalized energy spectrum is obtained by solving

$$
x^{4}=\frac{y^{5}}{1+2 y+y^{2}}
$$

where $x=k / k_{\Omega}$. The rate of energy transfer is given by

$$
\epsilon=C_{k}^{-3 / 2} \frac{k U_{k}^{3}}{1+C_{3} / y}
$$

Comparing (18) with (2), we found that Zeman's corresponding expression of the time scale of the triple velocity correlation is

$$
\tau_{3}=C_{K}^{-3}\left[1+\frac{C_{3}}{y}\right]^{-1} \tau_{n l}
$$

where $C_{3}$ is a constant introduced by Zeman.

Under rapid rotation, Eq. (19) reduces to

$$
\tau_{3}(k) \rightarrow \frac{1}{C_{3} C_{K}^{3}} \frac{\tau_{\Omega}^{2}}{\tau_{n l}(k)} .
$$

Eq. (20) indicates that Zeman's time scale for the triad nonlinear interactions differs from the key assumption of Squire et al. that the correlation time of the nonlinear triadic interactions 
is directly proportional to the short time scale $\tau_{\Omega}=1 / \Omega$. Therefore, it appears that Zeman's basic assumption that leads to his energy spectrum is not supported by LES computation. Indeed, Zeman ${ }^{16}$ noted that a further assumption

$$
1+C_{4} y^{-1}=y^{-1 / 2}
$$

is needed to obtain the "-2" spectrum (Eq. 4) from Eqs. (17)-(19) $\left(C_{4}\right.$ is another constant introduced by Zeman).

Bershadskii et al. ${ }^{2 \tau}$ have proposed a $E(k) \propto \zeta^{2 / 3}|k|^{-7 / 3}$ spectral form for rotating turbulence with some observational supports from stratospheric turbulence. Here $\zeta$ is the rate of spontaneous helicity generation. This spectral form was originally obtained by Brissand $e t$ $a l .{ }^{28}$ for the case of three dimensional turbulence, Bershadskii et $a l . .^{2 \tau}$ argued that the quasitwo-dimensional turbulence regime arises as a result of a spontaneous breaking of reflexional symmetry, which in turn is a consequence of the instability of two dimensional turbulence to three dimensional helical traveling waves and solitons.

\section{Conclusions}

The strong similarity between the MHD turbulence and isotropic turbulence subject to rotation has been noted. The MHD phenomenologies of Kraichnan ${ }^{4}$ and Matthaeus and Zhou ${ }^{15}$ were applied to rotating turbulence. When the turbulence is subject to strong rotation, an energy spectrum, $E(k)=C_{\Omega}(\Omega \epsilon)^{1 / 2} k^{-2}$, where the constant $C_{\Omega}=1.22-1.87$ is found. A hypothesis on the triple correlation decay rate, Eq. (7), leads to the spectral law given in Eqs. (9)-(11), which varies between the ' $-5 / 3$ ' (without rotation) and ' -2 ' laws (with strong rotation). We deduced a 'rule' (Eq. 8) that relates spectral transfer times to the eddy turnover time and the time scale for decay of the triple correlations. For intermediate rotation rate, the spectrum varies according to the value of a dimensionless parameter that measures the strength of the rotation wavenumber $k_{\Omega}=\left(\Omega^{3} / \epsilon\right)^{1 / 2}$ relative to the wavenumber $k$. An eddy viscosity is derived which explicitly depends on the rotation rate. 


\section{Acknowledgments}

The author would like to thank Professor C.G. Speziale for his stimulaling remarks. Also, thanks to Dr. M. Hossain and Dr. $\Lambda$. Mahalov for their useful conversations.

\section{References}

1. A. N. Kolmogorov, "The local structure of turbulence in incompressible viscous fluid for very large Reynolds number". Dokl. Akad. Nark SSSSR, 30, 301 (1941).

2. G.K. Batchelor, The theory of homogeneous turbulence (C'ambridge Univ. Press, Cambridge, UK, 1953)

3. A.S. Monin and A.M. Yaglom, Statistical Fluid Merhanics (Ml'T Press, 1975), Vol. 2.

4. R.H. Kraichnan, Inertial range spectrum of hydromagnetic turbulence, Phys. Fluids $8,1385,(1965)$

5. S.C. Traugott, Influence of solid-body rotation on screen-produced turbulence, NACA Tech. Note 4135, (1958)

6. R.A. Wigeland and H.M. Nagib, Grid-generated turbulence with and without rotation about the streamwise direction, IIT Fluids and IIcat Tinnsfer Rep., R78-1, Illinois Inst. of Tech., Chicago, Illinois (1978)

7. I. Jacquin, O. Leuchter, (.. Cambon, and J. Mathicu, Homogeneous turbulence in the presence of rotation, J. Fluid Merh., 220, 1 (19990)

8. S.V. Veeravalli, An experimenta] study of the effects of rapid rotation turbulence, Center for Turbulence Rescarch Briefs, 1990, Stanford University-N $\Lambda$ SA Ames Research Center (1990)

9. J. Bardina, J. H. Ferziger, and R. S. Rogallo, Effect of rotation on isotropic turbulence: computation and modeling, J. Fluid Merh., 154, 321 (1985) 
10. C.G. Speziale, N.N. Mansour, and R.S. Rogallo, Decay of turbulence in a rapidly rotating frame, Proc. 1977 Center for Turbulence Research Summer Program, Stanford University-NASA Ames Research Center (1987)

11. N.N. Mansour, C. Cambon, and C.G. Speziale, Theoretical and computational study of rotating isotropic turbulence, in Studies in Turbulence, edited by T.B. Gatski, S. Sarkar, and C.G. Speziale, Springer-Verlag (1992)

12. M. Hossain, Reduction in the dimensionality of turbulence due to a strong rotation, Phys. Fluids 6, 1077 (1994)

13. K.D. Squires, J.R. Chasnov, N.N. Mansour, and C. Cambon, Investigation of the asymptotic state of rotating turbulence using LES, Center for Turbulence Research Briefs, 1993, Stanford University-NASA Ames Research Center (1993)

14. C. Cambon and L. Jacquin, Spectral approach to non-isotropic turbulence subject to rotation, J. Fluid Mech., 202, 295 (1989)

15. W.H. Matthaeus and Y. Zhou, Extended inertial range phenomenology of magnetohydrodynamic turbulence, Phys. Fluids B1, 1929 (1989)

16. O. Zeman, A note on the spectra and decay of rotating homogeneous turbulence, Phys. Fluids, 6, 3221 (1994)

17. R. Grappin, U. Frisch, J. Léorat, and A. Pouquet, Alvénic fluctuations as asymptotic states of MHD turbulence, Astron. Astrophys., 102, 6 (1982)

18. R. Grappin, A. Pouquet, and J. Léorat, Dependence of MHD turbulence spectra on the velocity field-magnetic field correlation, Astron. Astrophys., 126, 51 (1983)

19. N.N. Mansour, C. Cambon, and C.G. Speziale, Single point modeling of initially isotropic turbulence under uniform rotation, Center for Turbulence Research Briefs, 1991, Stanford University-NASA Ames Research Center (1991) 
20. H. Tennekes and J.L. Lumley, A First course in turbulence (MIT press, Cambridge, MA, 1972)

21. Y. Zhou, Interacting scales and energy transfer in isotropic turbulence, Phys. Fluids $A, \mathbf{5}, 2511(1993)$

22. V. Yakhot and S.A. Orszag, Renormalization group analysis of turbulence. I. Basic theory, J. Sci. Comput., 1, 3 (1986).

23. R. Rubinstein, and J. M. Barton, Nonlinear Reynolds stress model and the renormalization group. Phys. Fluids A, 2, 1472 (1990).

24. Y. Zhou, G. Vahala, and S. Thangam, Development of a recursive RNG based turbulence model. Phys. Rev. E, 49, 5195 (1994).

25. C.G. Speziale, Analytical methods for the development of Reynolds stress closures in turbulence, Ann. Rev. Fluid Mech., 23, 107 (1991).

26. T. B. Gatski, and C.G. Speziale, On explicit algebraic stress models for complex turbulent flows, J. Fluid Mech., 254, 59. (1993)

27. A. Bershadskii, E. Kit and A. Tsinober, Spontaneous breaking of reflexional symmetry in real quasi-two-dimensional turbulence: stochastic traveling waves and helical solitons in atmosphere and laboratory, Proc. R. Soc. Lond. A, 441, 147 (1993).

28. A. Brissaud, U. Frisch, J. Leorat, M. Lesieur and A. Mazure, Helicity cascades in fully developed isotropic turbulence, Physics of Fluids, 16, 1366 (1973). 


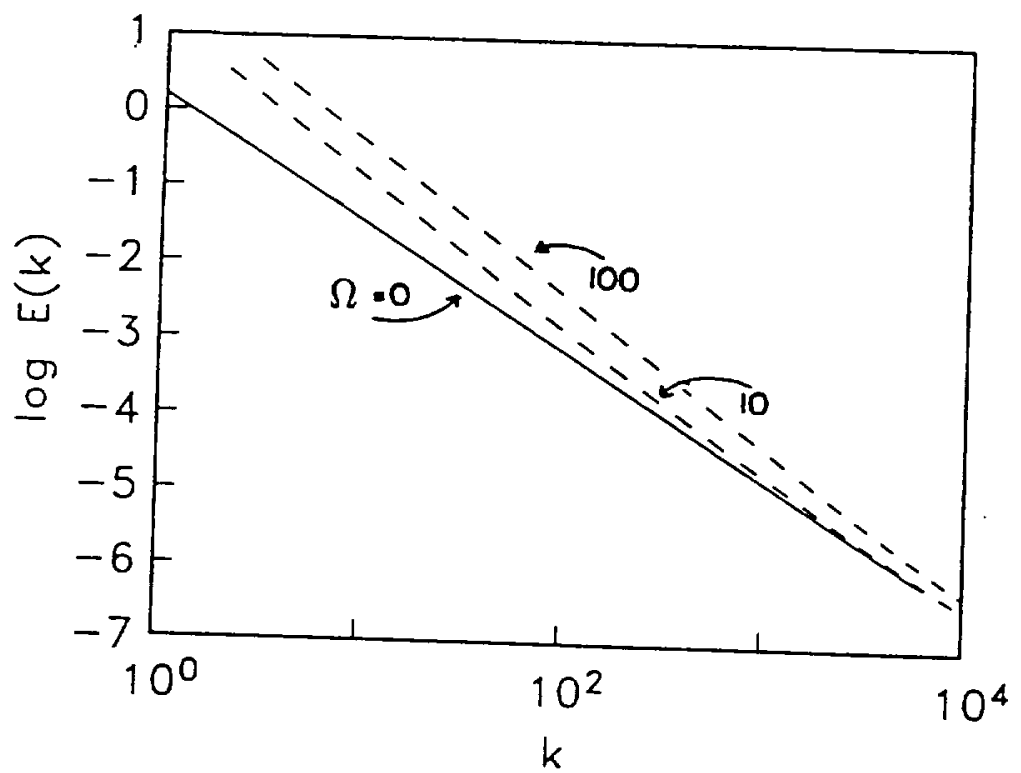

Fig. 1. Omnidirectional energy spectrum $E(k)$ versus wavenumber $k$, computed from Eqs. (9)-(11), for the case $A=0.7, \epsilon=1$, varying the rotation rate $\Omega=0,10,100$. There is a smooth transition from the Komogorov ' $-5 / 3$ ' case $\Omega=0$ to a spectrum $\Omega=100$ that approaches a ' -2 ' form. 
- 


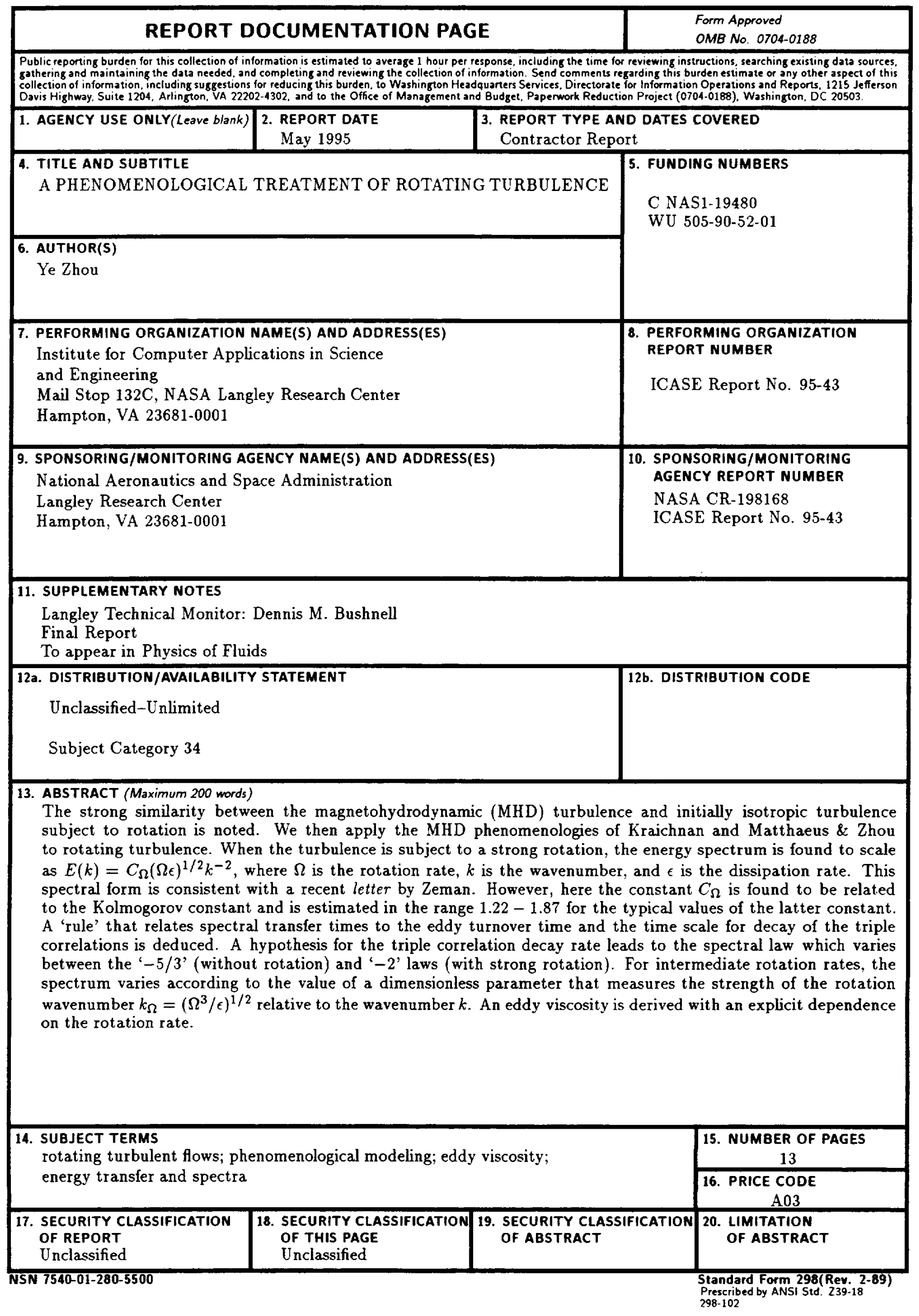


